\title{
PERIODIC ORBITS OF A PERTURBED 3-DIMENSIONAL ISOTROPIC OSCILLATOR WITH AXIAL SYMMETRY
}

\author{
JUAN L.G. GUIRAO ${ }^{1}$, JAUME LLIBRE ${ }^{2}$ AND JUAN A. VERA ${ }^{3}$
}

\begin{abstract}
We study the periodic orbits of a generalized Yang-Mills Hamiltonian $\mathcal{H}$ depending on a parameter $\beta$. This Hamiltonian consists of a 3 -dimensional isotropic harmonic oscillator plus a homogeneous potential of fourth degree having an axial symmetry, which implies that the third component $N$ of the angular momentum be constant. We prove that in each invariant space $\mathcal{H}=h>0$ the Hamiltonian system has at least 4 periodic solutions if either $\beta<0$, or $\beta=5+\sqrt{13}$; and at least 12 periodic solutions if $\beta>6$ and $\beta \neq 5+\sqrt{13}$. We also study the linear stability of these periodic solutions.
\end{abstract}

\section{INTRODUCTION AND STATEMENT OF THE MAIN RESULTS}

In this paper we study the periodic solutions of a generalized Yang-Mills Hamiltonian [13, 15], which consists of a 3-dimensional (or simply 3D) isotropic harmonic oscillator perturbed by a homogeneous potential of fourth degree

(1) $\mathcal{H}=\frac{1}{2}\left(p_{1}^{2}+p_{2}^{2}+p_{3}^{2}\right)+\frac{1}{2}\left(x_{1}^{2}+x_{2}^{2}+x_{3}^{2}\right)+\varepsilon\left(\left(x_{1}^{2}+x_{2}^{2}\right)^{2}+\beta\left(x_{1}^{2}+x_{2}^{2}\right) x_{3}^{2}\right)$.

Of course $\varepsilon$ is a small parameter. This perturbation exhibits an axial symmetry with respect to the $x_{3}$-axis which depends on one real parameters $\beta$, thus its study can be reduced to a family of Hamiltonian systems with 2 degrees of freedom fixing the third component of the angular momentum. In the paper [15] the authors studied two Hamiltonians whose motions take place in the plane $\left(x_{1}, x_{2}\right)$, one with a cubic potential and another with a quartic one, while we study a Hamiltonian in the space $\left(x_{1}, x_{2}, x_{3}\right)$ whose motion takes place outside the plane $\left(x_{1}, x_{2}\right)$ when the third component of the angular momentum is not zero. In [13] there is also studied the Yang-Mills Hamiltonian in the plane $\left(x_{1}, x_{2}\right)$.

When $p_{3}=x_{3}=0$ and the perturbation is $x_{1}^{2} x_{2}^{2}$ we obtain the called Contopoulos Hamiltonian, studied by him and coworkers during many years, see for instance $[5,6,7]$. The Contopoulos Hamiltonian studies the perturbed central part of an elliptical or barred galaxy without escapes. Several authors have been studied quartic homogeneous potentials (without quadratic terms), see for instance $[1,2,10]$. Some generalizations of the mechanical Yang-Mills Hamiltonian, with three, four or five quartic terms, have been considered in

2010 Mathematics Subject Classification. 70H12, 70H14, 70H33.

Key words and phrases. Periodic orbits, Averaging Theory, 3D isotropic oscillators, 3D Yang-Mills Hamiltonian, stability of periodic orbits. 
$[3,9,14,15]$. On the other hand, in [11] the authors study a similar problem but the perturbation in the Hamiltonian (1) is given by a cubic potential instead of a quartic one, and the tools there used are essentially based in the normal form theory while our tools are based in the averaging theory for studying periodic solutions.

The Hamiltonian (1) can be written in the called nodal-Lissajous variables $(l, g, \nu, L, G, N)$ (see section 2 for details), where $N=x_{1} p_{2}-x_{2} p_{1}$ is the first integral given by the third component of the angular momentum, and $G=\|\mathbf{x} \times \mathbf{p}\|$ is the first integral given by the total angular momemtum, where $\mathbf{x}=\left(x_{1}, x_{2}, x_{3}\right)$ and $\mathbf{p}=\left(p_{1}, p_{2}, p_{3}\right)$. In these variables the Hamiltonian (1) writes

$$
\mathcal{H}=L+\varepsilon \mathcal{P}(L, G, N, l, g) .
$$

Our main result on the periodic orbits of the Hamiltonian system associated to the Hamiltonian (1) is the following.

Theorem 1. For the Hamiltonian system associated to the Hamiltonian (1) or (2) the following statements hold.

(a) Assume that $\mathcal{H}=h>0$ and $N=0$. Then if $\beta \in(-\infty, 0) \cup(6,+\infty)$ there exist four $2 \pi$-periodic solutions $(L(l, \varepsilon), G(l, \varepsilon), g(l, \varepsilon))$ in the angular variable $l$ such that

$$
(L(0, \varepsilon), G(0, \varepsilon), g(0, \varepsilon)) \rightarrow\left(h, G_{0}, g_{0}\right) \quad \text { when } \varepsilon \rightarrow 0,
$$

where

$$
G_{0}=\frac{h \sqrt{\beta(\beta-6)}}{|\beta-3|}, \quad g_{0}=0, \frac{\pi}{2}, \pi, \frac{3 \pi}{2} .
$$

The two periodic orbits bifurcating from the two polar elliptic orbits of the $3 D$ isotropic harmonic oscillator with initial conditions $\left(h, G_{0}, 0\right)$ and $\left(h, G_{0}, \pi\right)$ are linearly stable if $\beta \geq 6$, and unstable if $\beta<0$. The two periodic orbits bifurcating from the two polar elliptic orbits of the $3 D$ isotropic harmonic oscillator with initial conditions $\left(h, G_{0}, \pi / 2\right)$ and $\left(h, G_{0}, 3 \pi / 2\right)$ are unstable if $\beta \geq 6$, and linearly stable if $\beta<0$.

(b) Assume that $\mathcal{H}=h>0$ and

$$
N=n= \pm \frac{2 h \sqrt{3(\beta-2)(\beta-6)}}{|\beta(\beta-4)|} \neq 0 .
$$

If $\beta>6$ and $\beta \neq 5+\sqrt{13}$ there exist four $2 \pi-$ periodic solutions $(L(l, \varepsilon), G(l, \varepsilon), g(l, \varepsilon))$ in the angular variable $l$ such that

$$
(L(0, \varepsilon), G(0, \varepsilon), g(0, \varepsilon)) \rightarrow\left(h, G_{0}, g_{0}\right) \quad \text { when } \varepsilon \rightarrow 0,
$$

where

$$
G_{0}=\sqrt{\beta-1}|n|, \quad g_{0}=0, \frac{\pi}{2}, \pi, \frac{3 \pi}{2} .
$$

The two periodic orbits bifurcating from the two inclined elliptic orbits of the $3 D$ isotropic harmonic oscillator with initial conditions $\left(h, G_{0}, 0\right)$ and $\left(h, G_{0}, \pi\right)$ are linearly stable, and the two periodic orbits bifurcating 
from the inclined elliptic orbits of the $3 D$ isotropic harmonic oscillator with initial conditions $\left(h, G_{0}, \pi / 2\right)$ and $\left(h, G_{0}, 3 \pi / 2\right)$ are unstable.

Theorem 1 is proved in section 3 .

From Theorem 1 given $h>0$ we have four periodic solutions for each of the following values

$$
n=0 \quad \text { and } \quad n= \pm \frac{2 h \sqrt{3(\beta-2)(\beta-6)}}{|\beta(\beta-4)|},
$$

when $\beta>6$ and $\beta \neq 5+\sqrt{13}$. While we have only four periodic solutions when $n=0$ and either $\beta<0$, or $\beta=5+\sqrt{13}$. In short we have the following result.

Corollary 2. The Hamiltonian system associated to the Hamiltonian (1) or (2) has at every energy level $\mathcal{H}=h>0$

(a) at least 4 periodic solutions if either $\beta<0$, or $\beta=5+\sqrt{13}$.

(b) at least 12 periodic solutions if $\beta>6$ and $\beta \neq 5+\sqrt{13}$.

In Celestial Mechanic the periodic orbits having the third component of the angular momentum $N$ equal to zero are usually called polar periodic orbits. Let $\gamma$ be a periodic orbit for which $N$ and $G$ are constant. Then the angle $I$ defined as $\cos I=N / G$ with $0 \leq I \leq \pi$ is called the inclination angle of $\gamma$. So, when $I$ is $\pi / 2$ the periodic orbit $\gamma$ is polar. When $I \in(0, \pi)$ the periodic orbit $\gamma$ is called inclined. Finally, when $I$ is zero or $\pi$ the periodic orbit is called equatorial. See Figures 1 and 2 respectively.

For proving Theorem 1 we use in an essential way Corollary 4 given in section 2. Thus with this corollary we can study the cases $|N|<G$ of Theorem 1, i.e. Theorem 1 studies polar and inclined periodic orbits of the Hamiltonian system associated to the Hamiltonian (1). But unfortunately the case $|N|=G$, i.e. the equatorial periodic orbits, cannot be studied using the nodalLissajous variables used in this paper.

We remark that the inclined periodic orbits found in the statement (b) of Theorem 1 have inclination angle $I$ satisfying $\cos I<1 / \sqrt{5}$. The angle $I=$ $63^{\circ} .43$.. for which $\cos I=1 / \sqrt{5}$ coincides with the so called critical inclination angle in the satellite theory, see for more details [4].

In section 2 we recall some basic results that we shall need for proving Theorem 1.

\section{Preliminary RESUlts}

In this section we first wrote our Hamiltonian system associated to the Hamiltonian (1) in the action-angle variables called nodal-Lissajous variables, and after we apply to the Hamiltonian system in these last variables a result of [12] (see the next Theorem 3), for obtaining the next Corollary 4, which will play a key role in the proof of our main result (Theorem 1). 


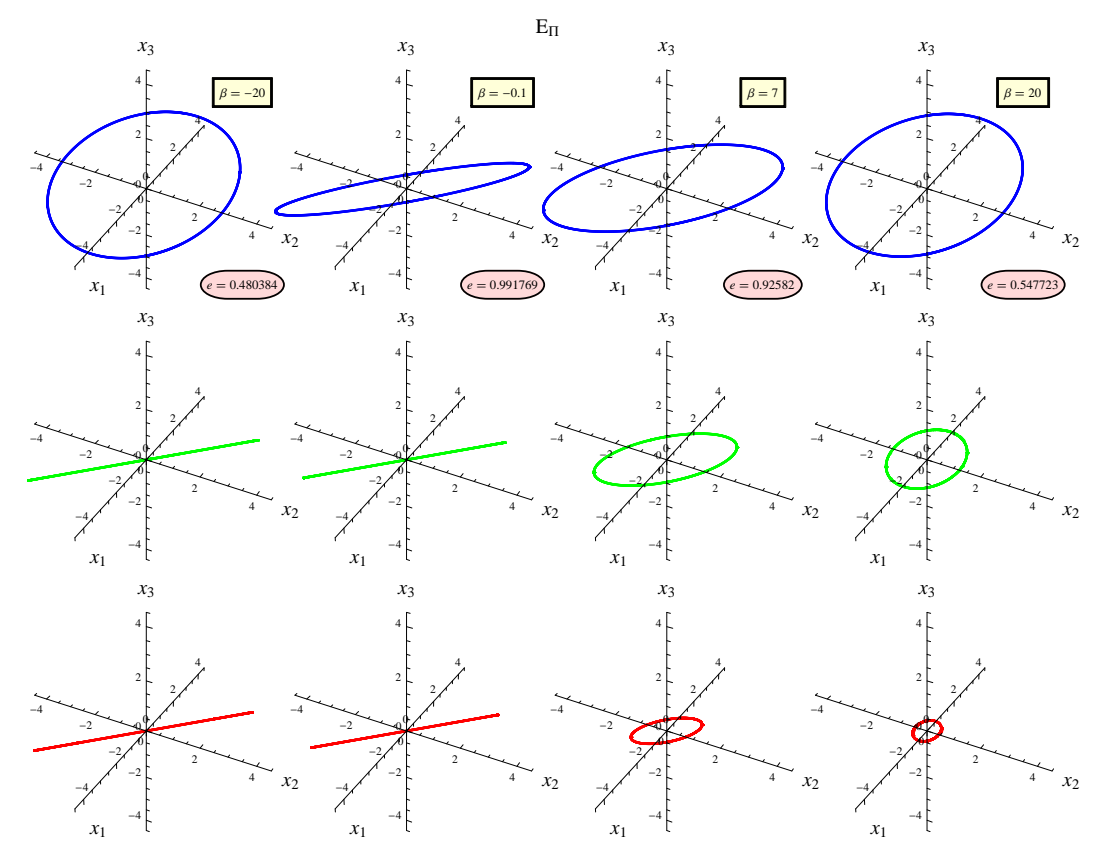

FiguRE 1. $E_{\pi}$ : The periodic orbits in the same column have the same values of the parameter $\beta$, in each column only change the value of the parameter $\varepsilon$, and each column correspond to one of the four families of periodic orbits of statement (a). All these periodic orbits correspond to the ones of statement (a) of Theorem 1. The green and red graphics correspond to periodic orbits for $\varepsilon=10^{-8}$ and $10^{-9}$ respectively. The value of $n=0$ and the computations has been done for $h=10$.

In this paper we study the periodic orbits of the Hamiltonian systems associated to Hamiltonians of the form

$$
\mathcal{H}=\frac{1}{2}\left(p_{1}^{2}+p_{2}^{2}+p_{3}^{2}\right)+\frac{\omega^{2}}{2}\left(x_{1}^{2}+x_{2}^{2}+x_{3}^{2}\right)+\varepsilon \mathcal{P}_{1}\left(x_{1}^{2}+x_{2}^{2}, x_{3}\right)
$$

using as main tool the next Theorem 3 , based in the averaging theory. When we apply the results that we shall obtain for the Hamiltonian (4) to Hamiltonian (1) we must take $\omega=1$.

The perturbations $\mathcal{P}_{1}\left(x_{1}^{2}+x_{2}^{2}, x_{3}\right)$ of the $3 \mathrm{D}$ isotropic harmonic oscillator that we consider in this work are invariant under the axial $\mathbb{S}^{1}$ action

$$
\begin{aligned}
\rho: \mathbb{S}^{1} \times T \mathbb{R}^{3} & \rightarrow T^{*} \mathbb{R}^{3} \\
(\theta,(\mathbf{x}, \mathbf{p})) & \mapsto\left(R_{\theta} \mathbf{x}, R_{\theta} \mathbf{p}\right)
\end{aligned}
$$




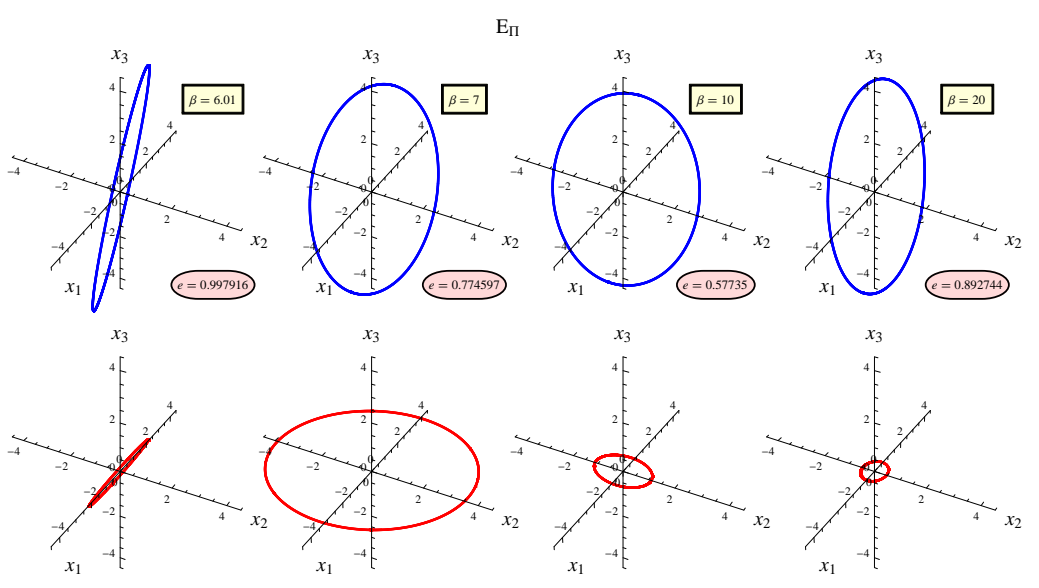

Figure 2. $E_{\pi}$ : The periodic orbits in the same column have the same values of the parameter $\beta$, in each column only change the value of the parameter $\varepsilon$, and each column correspond to one of the four families of periodic orbits of statement (b). All these periodic orbits correspond to the ones of statement (b) of Theorem 1. The red periodic orbits correspond to periodic orbits for $\varepsilon=10^{-9}$. The value of $n$ is given by the formula (3) and the computations has been done for $h=10$.

where

$$
R_{\theta}=\left(\begin{array}{ccc}
\cos \theta & -\sin \theta & 0 \\
\sin \theta & \cos \theta & 0 \\
0 & 0 & 1
\end{array}\right)
$$

Note that $R_{\theta}$ stands for the rotation about the third coordinate axis. From Noether's theorem, or by direct verification, can be obtained that the third component $N=x_{1} p_{2}-x_{2} p_{1}$ of the angular momentum is an integral of motion. This symmetry is a key point for simplifying the analysis of the family of Hamiltonian systems (4).

First we assume that the total angular momentum $G=\|\mathbf{x} \times \mathbf{p}\|$ does not vanish. This allows to choose the angular momentum vector $\mathbf{x} \times \mathbf{p}$ as the third coordinate axis. In the new coordinates the motion in the configuration space takes place in the $\left(Q_{1}, Q_{2}\right)$-plane, in particular we have $G=\left|Q_{1} P_{2}-Q_{2} P_{1}\right|$.

The Whittaker transformation (see Whittaker [18, Ch. 13, p. 343], and also Deprit[8])

$$
\begin{aligned}
\mathcal{W}:\left\{(\mathbf{x}, \mathbf{p}) \in T \mathbb{R}^{3}:|N|<G\right\} & \rightarrow T \mathbb{R}^{2} \times T^{*} \mathbb{S}^{1} \\
\left(x_{1}, x_{2}, x_{3}, p_{1}, p_{2}, p_{3}\right) & \mapsto\left(Q_{1}, Q_{2}, P_{1}, P_{2}, \nu, N\right)
\end{aligned}
$$


is given by

$$
\begin{aligned}
& \left(x_{1}, x_{2}, x_{3}\right)=\left(Q_{1} \cos \nu-Q_{2} \sin \nu \cos I, Q_{1} \sin \nu+Q_{2} \cos \nu \cos I, Q_{2} \sin I\right), \\
& \left(p_{1}, p_{2}, p_{3}\right)=\left(P_{1} \cos \nu-P_{2} \sin \nu \cos I, P_{1} \sin \nu+P_{2} \cos \nu \cos I, P_{2} \sin I\right),
\end{aligned}
$$

where $I$ is the inclination angle defined in section 1 .

The angle $\nu$ determines the nodal line, i.e. $\nu$ measures the angle from the $x_{1}$-axis to the straight line intersection between the $\left(x_{1}, x_{2}\right)$-plane and the $\left(Q_{1}, Q_{2}\right)$-plane. Thus $I$ is the angle between these two planes. The condition $|N|<G$ ensures that $\mathbf{x} \times \mathbf{p}$ is not parallel to the $x_{3}$-axis, so $\nu$ is well-defined.

The Whittaker transformation is a canonical transformation, i.e. the symplectic structure remains the standard one. In the new coordinates the Hamiltonian (4) becomes

$$
\mathcal{H}=\frac{1}{2}\left(P_{1}^{2}+P_{2}^{2}\right)+\frac{\omega^{2}}{2}\left(Q_{1}^{2}+Q_{2}^{2}\right)+\varepsilon \mathcal{P}\left(Q_{1}^{2}+Q_{2}^{2} \cos ^{2} I, Q_{2} \sin I\right) .
$$

By construction $\nu$ is a cyclic variable, thus the conjugate momentum $N$ is a first integral of the corresponding Hamiltonian system. For a fixed value $n$ of $N$ we have reduced the original system to a system of two degrees of freedom. The Whittaker transformation is only defined on the open and dense set of the phase space where $|N|<G$.

Now we shall write the Hamiltonian (5) in the Lissajous variables defined by

$$
\begin{aligned}
\mathcal{L}:\left\{(\mathbf{Q}, \mathbf{P}) \in T \mathbb{R}^{2}: G<L\right\} \times T \mathbb{S}^{1} & \rightarrow T \mathbb{T}^{3} \\
\left(Q_{1}, Q_{2}, P_{1}, P_{2}, \nu, N\right) & \mapsto(l, g, \nu, L, G, N)
\end{aligned}
$$

where

$$
\begin{aligned}
& Q_{1}=\sqrt{\frac{L+G}{2 \omega}} \cos (g+l)-\sqrt{\frac{L-G}{2 \omega}} \cos (g-l), \\
& P_{1}=-\omega\left(\sqrt{\frac{L+G}{2 \omega}} \sin (g+l)+\sqrt{\frac{L-G}{2 \omega}} \sin (g-l)\right), \\
& Q_{2}=\sqrt{\frac{L+G}{2 \omega}} \sin (g+l)-\sqrt{\frac{L-G}{2 \omega}} \sin (g-l), \\
& P_{2}=\omega\left(\sqrt{\frac{L+G}{2 \omega}} \cos (g+l)+\sqrt{\frac{L-G}{2 \omega}} \cos (g-l)\right) .
\end{aligned}
$$

These variables were introduced by Deprit in [8]. The angle $l$ describes the position on an ellipse at the configuration space, measured from its semiminor axis. So $l$ is named elliptic anomaly, which measures the position on the ellipse from its semi-minor axis. The other variables define this ellipse, which is centered at the origin of coordinates. The angle $g$ gives the position of the semi-minor axis reckoning from the $Q_{1}$-axis, i.e. from the nodal line. 
The eccentricity of the ellipse is given by

$$
e=\sqrt{\frac{2 \sqrt{L^{2}-G^{2}}}{L+\sqrt{L^{2}-G^{2}}}},
$$

and determines its shape, while the size is encoded in the length of its semiminor axis $b$ given by

$$
b=\sqrt{\frac{1}{\omega}\left(L-\sqrt{L^{2}-G^{2}}\right)} .
$$

The condition $G<L$ ensures that the ellipse does not degenerate to a circle, thus the angles $g$ and $l$ are well-defined. Finally $\nu$ still represents the angle of the ascending node of the orbital plane, the inclination of this plane with respect to the angular momentum vector is given by the angle $I$.

The transformation given by $\mathcal{L} \circ \mathcal{W}$ is known as the nodal-Lissajous transformation. The nodal-Lissajous variables are defined on $\left\{(\mathbf{x}, \mathbf{p}) \in T \mathbb{R}^{3}\right.$ : $|N|<G<L\}$. The Hamiltonian (5) expressed in the nodal-Lissajous variables has the form

$$
\mathcal{H}=\omega L+\varepsilon \mathcal{P}(L, G, N, l, g),
$$

where $\mathcal{P}(L, G, N, l, g)$ is the perturbing function $\mathcal{P}_{1}\left(x_{1}^{2}+x_{2}^{2}, x_{3}\right)$ expressed in the nodal-Lissajous variables. Since $\mathcal{H}$ and $N$ are first integral we shall restrict our attention to the invariant sets $\mathcal{H}=h>0$ and $N=n$ under the flow of the Hamiltonian systems associated to (4), (5) or (6). by

The Hamiltonian (6) belongs to a more general class of Hamiltonians given

$$
\mathcal{H}\left(I_{1}, I_{2}, \theta_{1}, \theta_{2}\right)=\mathcal{H}_{0}\left(I_{1}\right)+\varepsilon \mathcal{H}_{1}\left(I_{1}, I_{2}, \theta_{1}, \theta_{2}\right),
$$

where $\left(I_{i}, \theta_{i}\right)$ are the action-angle variables and $\varepsilon$ is a small parameter.

As usual the Poisson bracket of the functions $f\left(I_{1}, I_{2}, \theta_{1}, \theta_{2}\right)$ and $g\left(I_{1}, I_{2}, \theta_{1}, \theta_{2}\right)$ is computed by

$$
\{f, g\}=\sum_{i=1}^{2}\left(\frac{\partial f}{\partial \theta_{i}} \frac{\partial g}{\partial I_{i}}-\frac{\partial f}{\partial I_{i}} \frac{\partial g}{\partial \theta_{i}}\right) .
$$

The next result proved using the averaging theory for studying the periodic solutions of a differential system provides sufficient conditions for computing periodic orbits of the Hamiltonian system associated to the Hamiltonian (7). For more details on averaging theory see the books $[16,17]$.

Theorem 3 (See Theorem 1 of [12]). We define

$$
\left\langle\mathcal{H}_{1}\right\rangle=\frac{1}{2 \pi} \int_{0}^{2 \pi} \mathcal{H}_{1}\left(I_{1}, I_{2}, \theta_{1}, \theta_{2}\right) d \theta_{1},
$$


and we consider the differential system

$$
\begin{aligned}
& \frac{d I_{2}}{d \theta_{1}}=\varepsilon \frac{\left\{I_{2},\left\langle\mathcal{H}_{1}\right\rangle\right\}}{\mathcal{H}_{0}^{\prime}\left(\mathcal{H}_{0}^{-1}(h)\right)}=\varepsilon f_{1}\left(I_{2}, \theta_{2}\right), \\
& \frac{d \theta_{2}}{d \theta_{1}}=\varepsilon \frac{\left\{\theta_{2},\left\langle\mathcal{H}_{1}\right\rangle\right\}}{\mathcal{H}_{0}^{\prime}\left(\mathcal{H}_{0}^{-1}(h)\right)}=\varepsilon f_{2}\left(I_{2}, \theta_{2}\right),
\end{aligned}
$$

restricted to the energy level $H=h$ with $h \in \mathbb{R}$. The value $h$ is such that the function $\mathcal{H}_{0}^{-1}$ is a neighborhood of $h$ is a diffeomorphism. System (8) is a Hamiltonian system with Hamiltonian $\varepsilon\left\langle\mathcal{H}_{1}\right\rangle$. If $\varepsilon \neq 0$ is sufficiently small then for every equilibrium point $p=\left(I_{2}^{0}, \theta_{2}^{0}\right)$ of system (8) satisfying that

$$
\operatorname{det}\left(\left.\frac{\partial\left(f_{1}, f_{2}\right)}{\partial\left(I_{2}, \theta_{2}\right)}\right|_{\left(I_{2}, \theta_{2}\right)=\left(I_{2}^{0}, \theta_{2}^{0}\right)}\right) \neq 0,
$$

there exists a $2 \pi$-periodic solution $\gamma_{\varepsilon}\left(\theta_{1}\right)=\left(I_{1}\left(\theta_{1}, \varepsilon\right), I_{2}\left(\theta_{1}, \varepsilon\right), \theta_{2}\left(\theta_{1}, \varepsilon\right)\right)$ of the Hamiltonian system associated to the Hamiltonian (7) taking as independent variable the angle $\theta_{1}$ such that $\gamma_{\varepsilon}(0) \rightarrow\left(\mathcal{H}_{0}^{-1}(h), I_{2}^{0}, \theta_{2}^{0}\right)$ when $\varepsilon \rightarrow 0$. The stability or instability of the periodic solution $\gamma_{\varepsilon}\left(\theta_{1}\right)$ is given by the linear stability or instability of the equilibrium point $p$ of system (8). In fact, the equilibrium point $p$ has the linear stability behavior of the Poincaré map associated to the periodic solution $\gamma_{\varepsilon}\left(\theta_{1}\right)$.

We define

$$
\langle\mathcal{P}\rangle=\frac{1}{2 \pi} \int_{0}^{2 \pi} \mathcal{P}(L, G, N, l, g) d l .
$$

Corollary 4. For $\varepsilon \neq 0$ sufficiently small the Hamiltonian system defined by the Hamiltonian (6) in the invariant set $\mathcal{H}=h>0$ and $N=n \in \mathbb{R}$, has a $2 \pi$-periodic solution $\gamma_{\varepsilon}(l)=(L(l, \varepsilon), G(l, \varepsilon), g(l, \varepsilon))$ in the variable $l$ such that $(L(0, \varepsilon), G(0, \varepsilon), g(0, \varepsilon)) \rightarrow\left(\frac{h}{\omega}, G_{0}, g_{0}\right)$ when $\varepsilon \rightarrow 0$ where $p=\left(G_{0}, g_{0}\right)$ is a solution of the system

$$
\begin{aligned}
& f_{1}(G, g)=-\varepsilon \frac{1}{\omega} \frac{\partial\langle\mathcal{P}\rangle}{\partial g}=0, \\
& f_{2}(G, g)=\varepsilon \frac{1}{\omega} \frac{\partial\langle\mathcal{P}\rangle}{\partial G}=0
\end{aligned}
$$

satisfying that

$$
\operatorname{det}\left(\left.\frac{\partial\left(f_{1}, f_{2}\right)}{\partial(G, g)}\right|_{(G, g)=\left(G_{0}, g_{0}\right)}\right) \neq 0
$$


The stability or instability of the periodic solution $\gamma_{\varepsilon}(l)$ is given by the stability or instability of the equilibrium point $p$ of the differential system

$$
\begin{aligned}
\frac{d G}{d l} & =-\varepsilon f_{1}(G, g), \\
\frac{d g}{d l} & =\varepsilon f_{2}(G, g) .
\end{aligned}
$$

In fact, the equilibrium point $p$ has the stability behavior of the Poincaré map associated to the periodic solution $\gamma_{\varepsilon}(l)$.

Proof. The corollary follows directly from Theorem 3.

\section{Proof of Theorem 1}

We shall apply Corollary 4 to the Hamiltonian (4) with the perturbation given by

$$
\mathcal{P}_{1}\left(x_{1}^{2}+x_{2}^{2}, x_{3}\right)=\left(x_{1}^{2}+x_{2}^{2}\right)^{2}+\beta\left(x_{1}^{2}+x_{2}^{2}\right) x_{3}^{2}
$$

with $\beta \in \mathbb{R}$ and $\omega=1$, i.e. to the Hamiltonian (1).

Applying the nodal-Lissajous transformation to the Hamiltonian (1) we get that the function $\mathcal{P}(L, G, N, l, g)$ is

$$
\begin{aligned}
& \frac{1}{4}\left(\cos (2 I)(s \sin (g+l)-d \sin (g-l))^{2}+(s \sin (g+l)-d \sin (g-l))^{2}\right. \\
& \left.\quad+2(s \cos (g+l)-d \cos (g-l))^{2}\right)((1-\beta) \cos (2 I)(s \sin (g+l) \\
& \quad-d \sin (g-l))^{2}+(\beta+1)(s \sin (g+l)-d \sin (g-l))^{2} \\
& \left.\quad+2(s \cos (g+l)-d \cos (g-l))^{2}\right),
\end{aligned}
$$

where $s=\sqrt{L+G}, d=\sqrt{L-G}$ and $\cos I=N / G$. Therefore the function $\langle\mathcal{P}\rangle$ defined in (9) is

$$
\begin{aligned}
\frac{1}{64}( & -\left(d^{4}+4 d^{2} s^{2}+s^{4}\right)(4(\beta-5) \cos (2 I)+3(\beta-1) \cos (4 I)-7 \beta-41) \\
& +48\left(d^{2}+s^{2}\right) d s((\beta-1) \cos (2 I)+\beta-3) \sin ^{2}(I) \cos (2 g) \\
& \left.-48(\beta-1) d^{2} s^{2} \sin ^{4}(I) \cos (4 g)\right) .
\end{aligned}
$$

The functions $f_{1}(G, g)$ and $f_{2}(G, g)$ of the differential system (11) are

$$
\begin{aligned}
f_{1}(G, g) & =\frac{\Delta}{2 G^{4}}, \\
f_{2}(G, g) & =\frac{\Delta_{1}}{8 G^{5} \sqrt{L^{2}-G^{2}}}
\end{aligned}
$$


where

$$
\begin{aligned}
\Delta= & 3\left(G^{2}-N^{2}\right)\left(\sqrt{L^{2}-G^{2}}\left((\beta-1) N^{2}-G^{2}\right) L\right. \\
& \left.+(\beta-1)\left(G^{2}-L^{2}\right)\left(G^{2}-N^{2}\right) \cos (2 g)\right) \sin (2 g), \\
\Delta_{1}= & 6\left(G^{6}+\beta G^{4} N^{2}+G^{2}\left(-2 \beta L^{2} N^{2}-3(\beta-1) N^{4}\right)\right. \\
& \left.\quad+4(\beta-1) L^{2} N^{4}\right) L \cos (2 g) \\
& +\left(3(\beta-1) \cos (4 g)\left(G^{2}-N^{2}\right)\left(G^{4}+N^{2}\left(G^{2}-2 L^{2}\right)\right)\right. \\
& \quad-(\beta+3) G^{6}-3 G^{2} N^{2}\left(2(\beta+1) L^{2}+(\beta-1) N^{2}\right. \\
& \left.+18(\beta-1) L^{2} N^{4}\right) \sqrt{L^{2}-G^{2}},
\end{aligned}
$$

here $L=h$. The solutions $\left(G_{0}, g_{0}\right)$ of the system

$$
f_{1}(G, g)=0, \quad f_{2}(G, g)=0,
$$

give rise to periodic orbits for each $L=h>0$ and $N=n \in \mathbb{R}$ if the Jacobian $(10)$ at $\left(G_{0}, g_{0}\right)$ is non-zero, see Corollary 4.

Proof of statement (a) of Theorem 1. Assume that $N=0<G$ and $\mathcal{H}=h>$ 0 . Then it is not difficult to check that system (13) has the four solutions $\left(G_{0}, g_{i}\right)$ given by

$$
G_{0}=\frac{h \sqrt{\beta(\beta-6)}}{|\beta-3|}, \quad g_{i}=\frac{\pi}{2} i \quad \text { for } i=0,1,2,3 .
$$

For the solutions $\left(G_{0}, g_{i}\right)$ with $i=0,1$ we obtain

$$
\left.\frac{\partial\left(f_{1}, f_{2}\right)}{\partial(G, g)}\right|_{(G, g)=\left(G_{0}, g_{i}\right)}=\left(\begin{array}{cc}
0 & \frac{18 h^{2}(3-2 \beta)}{(\beta-3)^{2}} \\
\frac{(\beta-3)(18+\beta(\beta-6))}{36} & 0
\end{array}\right)
$$

for $\beta>6$, and

$$
\left.\frac{\partial\left(f_{1}, f_{2}\right)}{\partial(G, g)}\right|_{(G, g)=\left(G_{0}, g_{i}\right)}=\left(\begin{array}{cc}
0 & -\frac{18 h^{2} \beta}{(\beta-3)^{2}} \\
-\frac{18(3-2 \beta)}{(\beta-3)^{2}} & 0
\end{array}\right)
$$

for $\beta<0$. For the solutions $\left(G_{0}, g_{i}\right)$ with $i=2,3$ we obtain

$$
\left.\frac{\partial\left(f_{1}, f_{2}\right)}{\partial(G, g)}\right|_{(G, g)=\left(G_{0}, g_{i}\right)}=\left(\begin{array}{cc}
0 & -\frac{18 h^{2} \beta}{(\beta-3)^{2}} \\
-\frac{18(3-2 \beta)}{(\beta-3)^{2}} & 0
\end{array}\right)
$$

for $\beta>6$, and

$$
\left.\frac{\partial\left(f_{1}, f_{2}\right)}{\partial(G, g)}\right|_{(G, g)=\left(G_{0}, g_{i}\right)}=\left(\begin{array}{cc}
0 & \frac{18 h^{2}(3-2 \beta)}{(\beta-3)^{2}} \\
\frac{(\beta-3)(18+\beta(\beta-6))}{36} & 0
\end{array}\right)
$$

for $\beta<0$. 


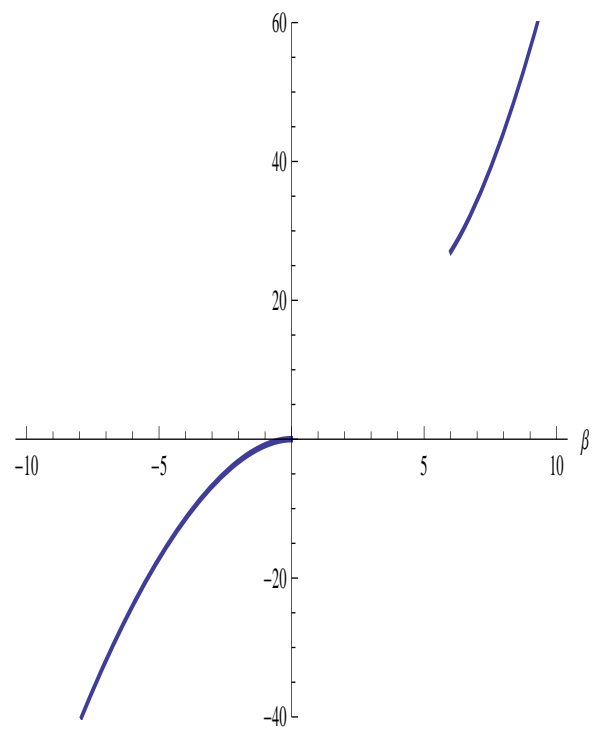

FiguRe 3. The graphics of $f_{1}(\beta, 1)$ for $\beta>6$ and of $f_{2}(\beta, 1)$ for $\beta<0$.

The Jacobian $(10)$ at $\left(G_{0}, g_{i}\right)$ for $i=0,2$ is

$$
J_{1}(\beta, h)=\frac{h^{2}(2 \beta-3)(18+\beta(\beta-6))}{2(\beta-3)}
$$

if $\beta>6$, and

$$
J_{2}(\beta, h)=-\frac{h^{2} \beta^{2}(\beta-6)}{2(\beta-3)}
$$

if $\beta<0$. The graphics of the functions $J_{i}(\beta, h)$ for $i=1,2$ are given in Figure 3. Therefore, by Corollary 4 the solutions $\left(G_{0}, g_{i}\right)$ for $i=0,2$ provide two periodic solutions linearly stable if $\beta>6$, and unstable if $\beta<0$.

On the other hand the Jacobian $(10)$ at $\left(G_{0}, g_{i}\right)$ with $i=1,3$ is

$$
J_{3}(\beta, h)=-\frac{h^{2} \beta^{2}(\beta-6)}{2(\beta-3)}
$$

if $\beta>6$, and

$$
J_{4}(\beta, h)=\frac{h^{2}(2 \beta-3)(18+\beta(\beta-6))}{2(\beta-3)}
$$

if $\beta<0$. The graphics of the functions $J_{i}(\beta, h)$ for $i=3,4$ are given in Figure 4. Hence the solutions $\left(G_{0}, g_{i}\right)$ for $i=1,3$ provide two periodic solutions linearly stable if $\beta<0$, and unstable if $\beta \geq 6$. This completes the proof of the statement (a) of Theorem 1. 


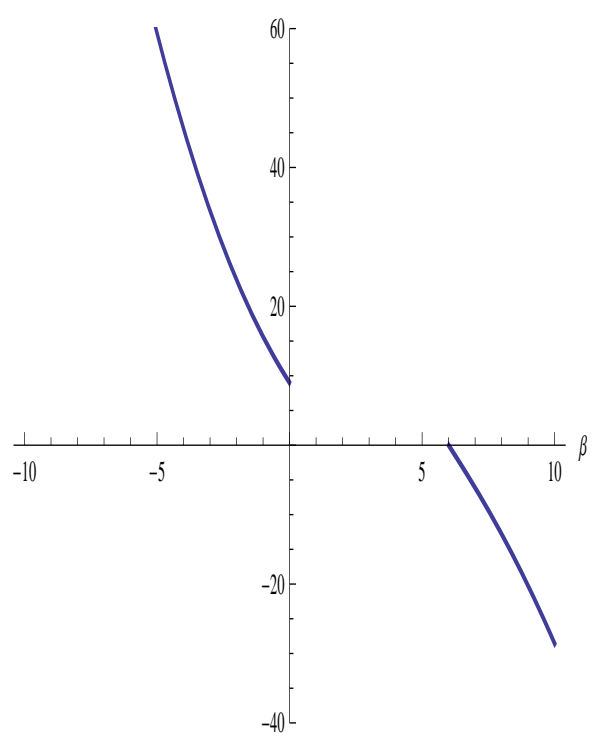

Figure 4. The graphics of $f_{3}(\beta, 1)$ for $\beta>6$ and of $f_{4}(\beta, 1)$ for $\beta<0$.

Proof of statement (b) of Theorem 1. Assume that $N=n \neq 0,|N|<G$ and $\mathcal{H}=h>0$. Then we can check that system (13) has the four solutions $\left(G_{0}, g_{i}\right)$ given by

$$
G_{0}=\sqrt{\beta-1}|n|, \quad g_{i}=\frac{\pi}{2} i \quad \text { for } i=0,1,2,3 .
$$

where

$$
n= \pm \frac{2 h \sqrt{3(\beta-2)(\beta-6)}}{|\beta(\beta-4)|} .
$$

For the solutions $\left(G_{0}, g_{i}\right)$ with $i=0,1$ we obtain

$$
\left.\frac{\partial\left(f_{1}, f_{2}\right)}{\partial(G, g)}\right|_{(G, g)=\left(G_{0}, g_{i}\right)}=\left(\begin{array}{cc}
0 & -\delta_{1} \\
\delta_{2} & 0
\end{array}\right)
$$

where

$$
\begin{aligned}
\delta_{1} & =\frac{3 h^{2}(\beta-2)^{2}(\beta(\beta-10)+12)^{2}}{(\beta-4)^{2}(\beta-1) \beta^{2}}, \\
\delta_{2} & =\frac{\beta(\beta-4)[\beta(\beta-4)[\beta(\beta(\beta-20)+88)-132]+864]}{4(\beta-6)(\beta-1)^{2}(\beta(\beta-10)+12)} .
\end{aligned}
$$

Moreover for the solutions $\left(G_{0}, g_{i}\right)$ with $i=2,3$ we obtain

$$
\left.\frac{\partial\left(f_{1}, f_{2}\right)}{\partial(G, g)}\right|_{(G, g)=\left(G_{0}, g_{i}\right)}=\left(\begin{array}{cc}
0 & \delta_{1} \\
\delta_{2} & 0
\end{array}\right) .
$$




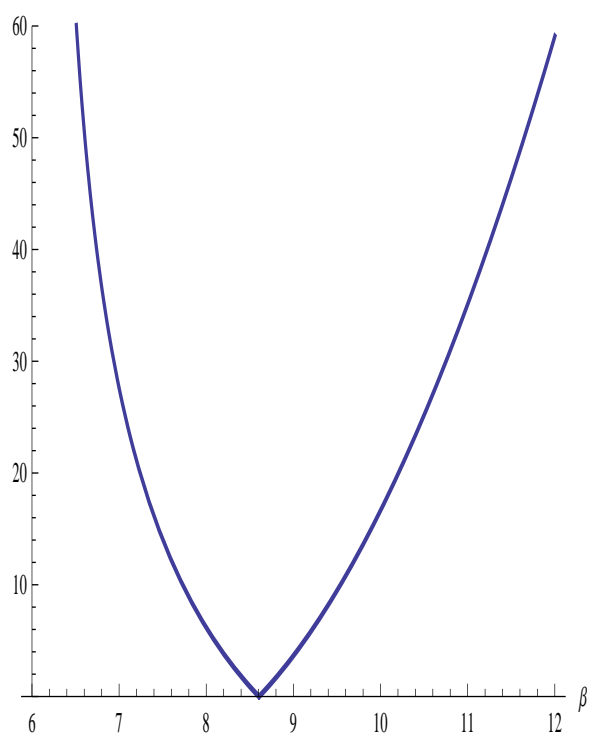

FiguRE 5. The graphic of $h_{1}(\beta, 1)$ for $\beta>6$.

The Jacobian $(10)$ at $\left(G_{0}, g_{i}\right)$ for $i=0,1$ is

$$
h_{1}(\beta, h)=\delta_{1} \delta_{2}
$$

with $\beta>6$. The graphic of the function $h_{1}(\beta, h)$ is given in Figure 5 . Therefore, by Corollary 4 the solutions $\left(G_{0}, g_{i}\right)$ for $i=0,1$ provide two linearly stable periodic solutions if $\beta>6$ and $\beta \neq 5+\sqrt{13}$, this last value of $\beta$ corresponds to the unique zero of the function $h_{1}(\beta, h)$ for $\beta>6$.

The Jacobian (10) at $\left(G_{0}, g_{i}\right)$ for $i=2,3$ is $g_{2}(\beta, h)=-\delta_{1} \delta_{2}$ with $\beta>6$. So, by Corollary 4 the solutions $\left(G_{0}, g_{i}\right)$ for $i=2,3$ provide two unstable periodic solutions if $\beta>6$ and $\beta \neq 5+\sqrt{13}$. This completes the proof of statement (b) of Theorem 1.

\section{Conclusion}

In this paper we have studied the periodic orbits of a Hamiltonian system defined by the Hamiltonian (1) being $\varepsilon$ a small parameter, this Hamiltonian is obtained perturbing the isotropic harmonic oscillator with a homogeneous potential of fourth degree. On the one hand such a Hamiltonian (1) is a generalization of the Yang-Mills Hamiltonian, and on the other hand that Hamiltonian also allows to study the motion of the central part of an elliptical or barred galaxy without escapes.

Our Hamiltonian system has two first integrals the Hamiltonian $\mathcal{H}$ and the third component of the angular momentum $N$. We prove that in each invariant space $\mathcal{H}=h>0$ the Hamiltonian system has at least 4 periodic solutions if 
either $\beta<0$, or $\beta=5+\sqrt{13}$; and at least 12 periodic solutions if $\beta>6$ and $\beta \neq 5+\sqrt{13}$. We also have studied the linear stability of these periodic solutions.

\section{ACKNOWLEDGEMENTS}

The first and third authors of this work are partially supported by MINECO/ FEDER grant number MTM2011-22587. The second author is partially supported by a MINECO/FEDER grant MTM2008-03437 and MTM2013-40998$\mathrm{P}$, an AGAUR grant number 2014SGR568, an ICREA Academia, the grants FP7-PEOPLE-2012-IRSES 318999 and 316338, FEDER-UNAB-10-4E-378.

\section{REFERENCES}

[1] M. Almeida, I. Moreira And F. Santos, On the Ziglin-Yoshida Analysis for some classes of homogeneous Hamiltonian systems, Braz. J. Phys. 28 (1998), 10.1590 S010397331998000400022.

[2] D. Biswas, M. Azam, Q. Lawande and S. Lawande, Existence of stable periodic orbits in the $x^{2} y^{2}$ potential: a semiclassical approach, J. Phys. A: Math. Gen. 25 (1992), 297-301.

[3] N. Caranicolas and H. Varvoglis, Families of periodic orbits in a quartic potential, Astron. Astrophys. 141 (1984), 383-388.

[4] S.L. Coffey, A. Deprit And B.R. Miller, The critical inclination in artificial satellite theory, Celestial Mech. 39 (1986), 365-406.

[5] G. Contopoulos, H. Papadaki and C. Polymilis, The structure of chaos in a potential without escapes, Celestial Mech. and Dyn. Astr. 60 (1994), 249-271.

[6] G. Contopoulos, M. Harssoula, N. Voglis and R. Dvorak, Destruction of islands of stability, J. Phys. A: Math. Gen. 32 (1999), 5213-5232.

[7] G. Contopoulos, C. Efthymiopoulos And A. Giongilli, Non-convergence of formal integrals of motion, J. Phys. A: Math. Gen. 36 (2003), 8639-8660.

[8] A. Deprit, The Lissajous transformation I. Basic, Celestial Mech. Dynam. Astronom. 51 (1991), 201-225.

[9] A. Elipe, J. Hietarinta And S. Tompadis, Comment on a paper by Kasperczuk, Cel. Mech. 58 (1994), 378-391, Celestial Mech. and Dyn. Astron. 62 (1995), 191-192.

[10] M. Falconi, E. Lacomba And C. Vidal, On the dynamics of mechanical systems with homogeneous polynomial potentials of degree 4, Bull. Braz. Math. Soc., New Series 38 (2007), 301-333.

[11] S. Ferrer, H. Hanssmann, J. Palacián and P. Yanguas, On perturbed oscillators in 1-1-1 resonance: the case of axially symmetric cubic potentials, J. Geom. Phys. 40 (2002), 320-369.

[12] J.L.G. Guirao, J. Llibre and J.A. Vera, Periodic orbits of Hamiltonian systems: Applications to perturbed Kepler problems, Chaos, Solitons \& Fractals 57 (2013), 105111.

[13] L. Jiménez-Lara And J. Llibre, Periodic orbits and non-integrability of generalized classical Yang-Mills Hamiltonian system, J. Math. Phys. 52 (2011), 032901-9 pp.

[14] S. Kasperczuc, Integrability of the Yang-Mills Hamiltonian System, Celestial Mech. and Dyn. Astr. 58 (1994), 387-391.

[15] A. Maciejewski, W. Radzki and S. Rybicki, Periodic trajectories near degenerate equilibria in the Henon-Heiles and Yang-Mills Hamiltonian systems, J. Dyn. Sys. and Diff. Eq. 17 (2005), 475-488. 
[16] J. A. Sanders F. Verhulst and J. Murdock, Averaging Methods in Nonlinear Dynamical Systems, Second edition, Applied Mathematical Sciences 59, Springer, New York, 2007.

[17] F. Verhulst, Nonlinear dierential equations and dynamical systems, Universitext, Springer, 1991.

[18] E. T. Whittaker, A Treatise on the Analytical Dynamics of Particles and Rigid Bodies, Cambridge Mathematical Library, Cambridge University Press, 1989.

${ }^{1}$ Departamento de Matemática Aplicada y Estadística. Universidad Politécnica de Cartagena, Hospital de Marina, 30203-Cartagena, Región de Murcia, SPAIN.

E-mail address: juan.garcia@upct.es

2 Departament de Matemàtiques. Universitat Autònoma de Barcelona, Bellaterra, 08193-Barcelona, Catalonia, Spain.

E-mail address: jllibre@mat.uab.cat

3 Centro Universitario de la Defensa. Academia General del Aire. Universidad Politécnica de Cartagena, 30720-Santiago de la Ribera, Región de Murcia, Spain.

E-mail address: juanantonio.vera@cud.upct.es 